\title{
Les inventions de la Grande Brière Mottière et de la « légende noire » briéronne (années 1770-années
} 1820)

The fabrications of the Grande Brière Mottière and the Brieronese black legend (1770s-1820s)

\section{Alain Gallicé}

\section{OpenEdition} Journals

\author{
Édition électronique \\ URL : https://journals.openedition.org/abpo/3442 \\ DOI : $10.4000 / a b p o .3442$ \\ ISBN : 978-2-7535-5365-7 \\ ISSN : 2108-6443 \\ Éditeur \\ Presses universitaires de Rennes
}

\section{Édition imprimée}

Date de publication : 30 décembre 2016

Pagination : 163-184

ISBN : 978-2-7535-5363-7

ISSN : 0399-0826

Référence électronique

Alain Gallicé, « Les inventions de la Grande Brière Mottière et de la « légende noire » briéronne (années 1770-années 1820) ", Annales de Bretagne et des Pays de l'Ouest [En ligne], 123-4 | 2016, mis en ligne le 30 décembre 2018, consulté le 21 septembre 2021. URL : http://journals.openedition.org/abpo/3442 ; DOI : https://doi.org/10.4000/abpo.3442 


\title{
Les inventions de la Grande Brière Mottière et de la "légende noire" briéronne (années 1770-années 1820)
}

\author{
Alain GALLICÉ \\ Docteur en histoire médiévale, chercheur associé au CRHIA, EA 1163, \\ université de Nantes - Président du conseil scientifique et de prospective \\ du Parc naturel régional de Brière
}

Le nom de Grande Brière Mottière a une histoire. Longtemps, le territoire a été inclus dans une zone plus vaste de marais désignée par le terme général de Brière (ou Brières ou Bruyère) et, afin de l'individualiser, il lui était accolé un qualificatif - " grande ${ }^{1}$ " - ou une périphrase faisant allusion à l'exploitation de la tourbe ${ }^{2}$. Puis, dans les années 1780, s'impose l'expression " Brière, motière et terreins contenant des tourbes et mottes à brûler " avant que, dans les années 1820, n'apparaisse l'expression actuelle qui s'institutionnalise progressivement avec la création de la Commission syndicale de Grande Brière Mottière ${ }^{3}$.

Ces mutations lexicales correspondent à deux temps forts de l'histoire briéronne au cours desquels le territoire a été jugé menacé : les années 1770-1780 sont marquées par l'affaire des Équibadeaux et un projet de dessèchement des marais de Donges afféagés par le vicomte de Donges et le seigneur de Besné à la compagnie de Bray; entre 1817 et 1825, le dessèchement des marais de Donges est réalisé par cette même compagnie.

Ces événements suscitent un fort mécontentement, une large mobilisation de l'opinion locale et régionale et une agitation qui génèrent des

1. OGÉe, Jean-Baptiste, Dictionnaire historique et géographique de la province de Bretagne; dédié à la nation bretonne, 4 vol., Nantes, Vatar, 1778-1780; nouv. éd. par MARTEVILLE, Alphonse et VARIN, Pierre, 2 vol., Rennes, Molliex, 1843-1845, t. II, p. 522, dit ce qualificatif de " grande " être de règle chez les habitants de Montoir.

2. Ainsi, sur les cartes de Cassini figure l'expression « Friches où l'on tire de la Tourbe ou Mottes".

3. Cette commission, premier organisme d'administration du territoire, est créée par l'ordonnance du 3 octobre 1838. Lors de sa première réunion, le 3 février 1839, elle s'intitule " Commission syndicale pour l'exploitation de la tourbe de la Grande Brière Mottière ", Arch. dép. de Loire-Atlantique, $1713 \mathrm{~S} 1$. 
actions nombreuses aux formes variées (publications de mémoires, instances judiciaires, voies de faits, voire émeutes...). Dans ce contexte, au cours des années 1770-1780, afin de trouver des soutiens, les défenseurs du territoire forgent une certaine image du territoire qui, cohérente et organisée, s'impose comme un stéréotype. En cela, nous pouvons parler d'invention ${ }^{4}$. Les événements des années 1820 contribuèrent à préciser certains points de cette représentation, mais surtout ils suscitèrent une autre " invention ", celle de la " légende noire " briéronne.

Le retour sur ces événements permet de comprendre comment se forgent les représentations des zones humides et l'identité de leurs habitants, représentations trop souvent attribuées à leur seule altérité. Après avoir étudié l'invention du territoire dans les années 1771-1780, en précisant son contexte, ses aspects, ses stéréotypes, ses acteurs et les raisons de sa réussite, nous envisagerons, au cours des années, entre 1817 et 1830, le devenir de cette image du territoire avant de présenter la légende noire briéronne, ses aspects, les raisons de sa mise en place et celles de son succès.

\section{L'invention de la " Brière, Mottière et terreins contenant des tourbes et mottes à brûler " (années 1770-1780)}

\section{Le contexte : un territoire jugé menacé}

Dans la seconde moitié du XVIII ${ }^{\mathrm{e}}$ siècle, deux affaires, en lien avec la Grande Brière Mottière, bien qu'indépendantes l'une de l'autre, s'entremêlent : celle des Équibadeaux (1754-1789) et celle du projet de dessèchement des marais de Donges (1771-1782) ${ }^{5}$.

Leurs liens avec la Grande Brière Mottière sont d'ordre différent. Dans l'affaire dite des Équibadeaux - du nom d'un terrain afféagé par le roi au seigneur de Saint-Denac -, ce lien est direct puisqu'il s'agit d'un empiétement perpétré par cet afféagiste sur la Grande Brière Mottière que lui contestent les riverains en arguant du statut de ce territoire. Précisons : ce territoire est un commun qui ne relève d'aucun seigneur et dont les

4. À l'image de l' "invention de la Bretagne ", voir Bertho, Catherine, "L'invention de la Bretagne. Genèse sociale d'un stéréotype ", dans L'identité, Actes de la recherche en sciences sociales, vol. 35, novembre 1980, p. 45-62.

5. Présentation de ces affaires dans GuIHAIRE, Albert, La Brière dans le droit coutumier, Rennes-Quimper, Impr. M. Simon/Librairie M. Le Dault, 1942, p. 144-174; GALLICÉ, Alain, « La défense de la Grande Brière Mottière dans la seconde moitié du XVIII ${ }^{\mathrm{e}}$ siècle : l'affaire des Équibadeaux, le projet de dessèchement des marais de Donges, et les lettres patentes du 28 janvier 1784 ", à paraître. Sur la question plus générale du dessèchement des marais, voir DiENNE, comte de, Histoire du dessèchement des lacs et marais en France avant 1789, Paris, Champion, 1891, ouvrage qui reste de référence; plus récemment pour le XVII ${ }^{\mathrm{e}}$ siècle, MORERA, Raphaël, L'assèchement des marais en France au XVIt siècle, Rennes, Presses universitaires de Rennes, 2011, qui renouvelle les perspectives; et encore BouRDE André J.,

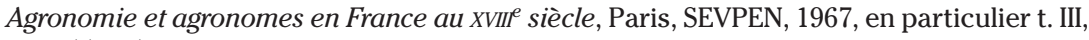
p. 1455-1457, pour le cas de la Brière. 
droits d'usage (attestés par un acte du duc François II du 8 août $1461^{6}$ ) ne sont pas réservés aux habitants d'une seule seigneurie mais sont partagés par l'ensemble des habitants des paroisses riveraines. Au terme d'un long combat juridique, ces droits d'usage sont assimilés à des droits de propriété par les lettres patentes du 28 janvier 1784 qui reconnaissent aux riverains la "propriété, possession et jouissance commune et publique " de la "Brière, Mottière et terreins contenant des tourbes et mottes à brûler ". Un tel statut est original par rapport au droit et à la rationalité juridique qui s'impose alors ${ }^{7}$ : en effet, fondée sur l'exercice d'anciens droits d'usage, cette propriété est collective et inaliénable puisqu'elle n'inclut ni le droit de vendre, ni celui de louer.

Quant au projet de dessèchement des marais de Donges, le lien avec la Grande Brière Mottière est indirect. En effet, la compagnie de Bray, constituée le 2 décembre 1771 pour réaliser leur dessèchement, a très rapidement exclu d'intervenir en Grande Brière Mottière. Cependant, ses adversaires lui prêtent des intentions cachées - dessèchement du territoire ou appropriation de l'exploitation et de la commercialisation de la tourbe - et, surtout, ils estiment que le dessèchement des marais de Donges menace la Grande Brière Mottière et ses productions. En effet, leur dessèchement, voire le simple approfondissement de la rivière du Brivet qui les traverse, entrâ̂nerait, prétendent-ils, celui de la Grande Brière Mottière et la disparition de ses productions. De fait, la Grande Brière Mottière est instrumentalisée par les opposants à la compagnie de Bray afin de s'assurer les plus larges soutiens. Dans cette perspective, ils ont, par leurs prises de position, brossé une certaine image du territoire ${ }^{8}$.

\section{La Grande Brière Mottière selon ses défenseurs}

\section{Un territoire doté d'un sol, d'une végétation et d'un sous-sol originaux}

Située sur le " territoire de Guérande ", " entre et dans les paroisses de Guérande, Saint-Lyphard, Saint-André-des-Eaux, Escoublac, Saint-Nazaire,

6. GAllicé, Alain, " Des “titres” médiévaux de la Grande Brière Mottière ou de l'histoire au mythe : "lettres patentes" du 8 août 1461 et "donation" d'Anne de Bretagne ", Bulletin de la Société archéologique et historique de la Loire-Atlantique et de Nantes, t. 150, 2015, p. 85-112.

7. Rappelons que selon le principe, " nulle terre sans seigneur ", même en l'absence de titres attestant la possession seigneuriale, tous communs ou terres vagues, vaines et décloses, sont attribuables à la seigneurie dans laquelle ils sont enclavés et que, toujours en l'absence de titres d'inféodation, si les droits d'usage sont exercés à titre gratuit par les vassaux (ce qui était le cas de la Grande Brière Mottière), le seigneur garde la propriété entière des terrains concernés et peut en disposer quitte, par un cantonnement, à dédommager les habitants de la perte de leurs droits de " communer".

8. Cette étude repose sur divers documents : Arch. dép. de Loire-Atlantique, C 111-112; ibidem, E, dépôt, 8, Guérande, DD 1-3, qui concernent soit l'affaire des Équibadeaux, soit le projet de dessèchement des marais de Donges, soit les deux à la fois, et qui souvent se répètent. Les citer tous alourdirait considérablement les notes de bas de page, aussi limitera-t-on les références à ceux dont sont extraites les citations retenues. 
Montoir, Donges, Crossac, Prinquiau, Pontchâteau et Herbignac ", occupant une "surface de 7 à 9 lieues ${ }^{9}$ ", la Grande Brière Mottière se distingue d'abord des zones voisines par la nature de son sol tourbeux.

Ce sol est constitué d'" un terrain spongieux, de médiocre épaisseur [4 pieds] composé de fibres boiseuses et de matière sulphureuse ». Aussi est-il " combustible et inflammable " et " le feu [y] prend presque tous les ans ", le réduisant en cendres. Un tel sol est inapte à la vie agricole. Mêlé à d'autres, il ne se mélange pas, s'assèche, reste inflammable et les " amaigrit ", sauf si la tourbe est préalablement réduite en cendre.

Un tel sol est couvert d'une végétation particulière. Les " avortes ", qui " ne croissent que dans la Brière ", sont des sortes de petits saules dont les feuilles et les fruits, pressés entre les doigts, dégagent " une odeur forte et agréable " et dont le bois est utilisé pour cuire le pain. Lorsque l'eau se retire, au sol ne se voient " guère que de mauvaises herbes ", dont les espèces, "propres à la seule tourbière ", forment " un tissu de plantes marines et filamenteuses dont il serait véritablement impossible d'extirper le germe ". La plupart sont " rousses, maigres et sèches " tel le lin de Brière. Cette végétation, qui " répugne aux bestiaux, les amaigrit et leur donne des poux ", est en lien avec la tourbe et la qualité de celle-ci. Aussi " tous ceux qui pratiquent la Brière connaissent, même avant de fouiller la terre, à la seule différence des mauvaises herbes de la surface, quelle est la qualité bonne ou médiocre du mottage ${ }^{10}$ ". Ainsi, " l'essence " de la Grande Brière Mottière est-elle la tourbe, et elle est sa seule ressource.

Mais l'originalité de ce territoire ne se limite pas à sa surface. Son sol est " soutenu sur un volume d'eau dont on ne trouve pas le fond ${ }^{11}$ " : on peut y introduire un bâton sans rencontrer de résistance, une perche de 20 pieds s'y enfoncerait toute entière et si l'on fait un trou, il se remplit "d'une eau fade mais bienfaisante qui s'élève en forme de jet d'eau et dont boivent les Briérons pendant leurs travaux ${ }^{12}$ ". Si le sol dessèche en surface, en profondeur subsiste toujours une " terre molle, détrempée, et sans résistance ${ }^{13}$ ". Dans ces conditions, établir des chemins est chose impossible : " une charrette ne pouvant pas s'engager dans l'ornière d'une autre qui y auroit déjà passé, sans courir les risques presque inévitables de se perdre " : " dix chevaux ne l'en tireroient point ". Le "bourbier de la Brière n'est susceptible d'aucun chemin fixe ", ce qui rend "indispensable

9. Arch. dép. de Loire-Atlantique, Guérande, dépôt 8, DD 3, " Mémoire ".

10. Arch. dép. de Loire-Atlantique, Guérande, dépôt 8, DD 3, 12 et 13 avril 1785, procèsverbal de délimitation des Équibadeaux.

11. Arch. dép. de Loire-Atlantique, C 112; ibidem, E, dépôt, 8, Guérande, DD 2, "Requête présentée au Roy en son Conseil par les vassaux bien tenants et habitans des paroisses dépendantes de la vicomté de Donges, savoir des paroisses de Donges, de Montoir, de Crossac, de Prinquiau et du bailliage de Crévy ".

12. Arch. dép. de Loire-Atlantique, E, dépôt, 8, Guérande, DD 2, procès-verbal de délimitation des Équibadeaux.

13. Arch. dép. de Loire-Atlantique, C 111, p. 16, procès-verbal de levée du plan figuratif des marais de Donges. 
que les bords de cette tourbière soient libres, pour pouvoir y porter et y amonceler les mottes ${ }^{14}$ ".

La spécificité de la Grande Brière Mottière est telle qu'"il y a plus de différence entre le terrain des Équibadeaux et la Brière qu'entre un lac et une montagne quoique l'un soit bas et l'autre élevé. Il y a plus de différence entre ces deux terrains qu'entre une falaise et une saline, quoique l'une soit toujours sablonneuse et l'autre nécessairement $\operatorname{argileuse}^{15}$ ".

\section{Un territoire sain}

Selon Emmanuel Olivaud, maître en chirurgie exerçant au bourg de Montoir, qui s'exprime le 11 juillet 1775, les populations locales sont sensibles " aux fièvres putrides et malignes ", mais il attribue celles-ci à "l'air de la mer " et non pas aux zones humides qui avoisinent le bourg qui sont naturellement saines. Cependant, cet état de choses est fragile et des travaux de dessèchement seraient cause d'une catastrophe sanitaire : "[le] fossoyement des terres pour l'excavation des canaux " libérerait " des substances putrides et malignes, ennemies de l'espèce humaine, provenant des amas de boue et autres matières fétides " et de la putréfaction des végétaux et d'animaux amoncelés par le temps, génératrices de maladies contagieuses et mortelles. Il se réfère à l'exemple des marais du haut Brivet, récemment desséchés, où règne " une fièvre putride et maligne qui tue presque tous les vieillards et beaucoup de jeunes personnes ${ }^{16}$ ".

Cependant, alors que la compagnie de Bray insiste à l'envi sur le gain économique et social à attendre du dessèchement, du côté des opposants, cette question de la salubrité du marais n'est guère exposée que par Olivaud, sans doute en raison de la prégnance des idées aéristes qui dominent à la fin du XVIII ${ }^{\mathrm{e}}$ siècle, en particulier dans l'administration pour qui dessèchement signifie progrès économique, amélioration sanitaire et mieux être social ${ }^{17}$.

\section{Un territoire doté par la Providence (ou la nature) de ressources spécifiques sans cesse renouvelées}

Ce milieu particulier est conçu comme un véritable écosystème. En effet, il " végète ", c'est-à-dire que ses ressources se renouvellent naturelle-

14. Arch. dép. de Loire-Atlantique, C 112, procès-verbal de levée du plan figuratif... ; ibidem, dépôt, 8, Guérande, DD 2, " Moyens précis, procuration des paroisses circonvoisines de la Brière".

15. Arch. dép. de Loire-Atlantique, E, dépôt, 8, Guérande, DD 2, "Requête au Conseil du roi des paroisses de Guérande, Saint-Lyphard et Saint-André-des-Eaux ", 16 mai 1777.

16. Arch. dép. de Loire-Atlantique, C 111, procès-verbal de levée du plan figuratif..., p. 119-120.

17. Entre autres, DEREX, Jean-Michel, "Pour une histoire des zones humides en France (XVII $-{ }^{\mathrm{e} I X}{ }^{\mathrm{e}}$ siècle). Des paysages oubliés, une histoire à écrire ", Histoire et sociétés rurales, $\mathrm{n}^{\mathrm{o}} 15 / 1,2001$, p. 17-21. 
ment. Si la régénération de la tourbe - qui intervient, dit-on, tous les « huit à dix ans " - est contestée par quelques-uns, et donne lieu à des explications différentes, elle est, à n'en pas douter, admise par les Briérons qui l'attribuent à la présence de l'eau en surface durant une partie de l'année et continuellement à une certaine profondeur. Un dessèchement " tarirait " la " source précieuse de sa formation et de son entretien ", la "dénaturerait " et l'exposerait à une destruction complète par incendie. Aussi la Grande Brière Mottière est-elle un système dont la cohérence ne peut avoir été pensée que par la Providence ${ }^{18}$. Il en découle que "La Brière ne peut être que la Brière ", qu'elle ne peut permettre " aucune autre culture " que celles dont elle dispose, et quand bien même pourrait-elle être convertie " en terre labourable ou en prairie ", le revenu serait infime, équivalant tout au plus au dixième de ce qu'il est, car elle offre " trop peu de liaison, de consistance et de fond " pour pouvoir donner de " vraies prairies " ou des terres cultivables ${ }^{19}$.

Une conclusion s'impose : " en un mot prétendre entreprendre de dessécher les marais, c'étoit vouloir être plus sage que la Providence qui avoit tout disposé et arrangé de la sorte pour les différents besoins du pays ${ }^{20}$ " en lui procurant des ressources spécifiques et en assurant leur continuelle reproduction.

\section{Un territoire aux ressources largement suffisantes pour assurer la vie des riverains ${ }^{21}$}

Cette nature particulière est généreuse. Sa principale ressource est la tourbe. Pour les " pauvres riverains de la Brière ", elle est la " seule récolte d'un grand nombre d'entre eux ". Son extraction leur assure le chauffage mais aussi les moyens de vivre car " ils vendent l'excédent de leurs provisions ". Présents sur les lieux durant l'été 1774, les commissaires des états de Bretagne évoquent des Briérons " zélés et laborieux journellement occupés à l'extirpation pénible des mottes ", alors que "les hommes coupent des mottes [...] leurs femmes et leurs enfants tout petits les portent et les tournent au soleil ». Ainsi, la vie des Briérons ne serait liée qu'à l'exploita-

18. Entre autres, Arch. dép. de Loire-Atlantique, C 111, procès-verbal de levée du plan figuratif..., p. 142-144; ibidem, E, dépôt, 8; Guérande, DD, 2 ; " Moyens précis, procuration des paroisses circonvoisines de la Brière ", " Mémoires ". "Requête présentée au Roy en son Conseil par les vassaux bien tenants et habitans des paroisses dépendantes de la vicomté de Donges... ".

19. Arch. dép. de Loire-Atlantique, C 112 et ibidem, E, dépôt, 8, Guérande, DD 25, "Moyens précis, procuration des paroisses circonvoisines de la Brière "; " Mémoires " de Rouaud de La Villemartin aux états de Bretagne.

20. Arch. dép. de Loire-Atlantique, C 111, procès-verbal de levée du plan figuratif..., p. 144.

21. Ce développement repose sur de très nombreux documents qui répètent à satiété les mêmes arguments, Arch. dép. de Loire-Atlantique, C 111, procès-verbal de levée du plan figuratif... ; ibidem, E, dépôt, 8, Guérande, DD 1, entre autres " Moyens précis, procuration des paroisses circonvoisines de la Brière ", " Mémoire "... 
tion de la tourbe et ne serait assurée que par son commerce. Sans ce " pain des riverains ", " 6 à 7000 personnes seraient réduites à mourir de faim, ou à s'expatrier, et 40 à 50000 seraient ruinées ".

Toutefois, certains n'omettent pas de mentionner que le territoire offre à ses riverains d'autres ressources : pâturage, litière et donc fumier, roseaux qui alimentent les fours et servent à la couverture de leurs maisons, portée par des charpentes faites parfois de mortas - arbres fossiles extraits du sous-sol tourbeux -, ce qui est une aubaine pour un territoire où le bois manque cruellement. Mais rares sont ceux qui signalent que les " terres de labour ", situées à l'intérieur des îles briéronnes, sont cultivées, sinon pour dire qu'elles n'assurent de quoi vivre que pour " un mois par an ". De même, l'usage des cendres de tourbe comme engrais et celui de la tourbe comme amendement des terres lourdes ne sont qu'épisodiquement évoqués. Quant à l'exploitation de l'osier, à la production de poterie ou du lin, et, surtout, à la pratique de la chasse et de la pêche, activités pourtant inhérentes aux zones humides, il n'en est pas fait état. Sans doute que chasse et pêche, dont les droits sont liés aux seigneuries, auraient pu conduire à poser la question du statut du territoire, ce qui n'est pas souhaité...

\section{Un territoire nécessaire aux lieux avoisinants et à la province ${ }^{22}$}

La tourbe fait l'objet d'un " commerce prodigieux ». Elle est " d'une nécessité absolue " pour "le grand territoire de Guérande ", où il n'y a pas ou peu bois de chauffage. La tourbe est également utilisée comme combustible dans toutes les paroisses de l'estuaire de la Loire, à Nantes, à La Roche-Bernard, à Redon, sur toute la côte jusqu'à Vannes et au-delà jusqu'à Lorient, à Port-Louis, à Belle-Île, à Brest et au sud de la Loire dans les îles de Bouin, d'Yeu, de Noirmoutier, de Ré et d'Oléron, ainsi qu'à Beauvoir, à Bourgneuf, à La Rochelle et à Bordeaux. Au total, dans tout le comté nantais, dans " la moitié du continent de la province de Bretagne ", dans " 42 paroisses de la côte de Bretagne et du Poitou ", la tourbe briéronne évite à 200000 personnes de " périr de froid et de misère ", et à 500000 autres d'être privées " des choses les plus nécessaires à la vie ", car sans elle, elles seraient dans "l'impossibilité de faire cuire leur pain, et bouillir leur pot " et souffriraient " une misère affreuse ". Ce combustible serait même le seul utilisé dans certaines villes (Nantes, Guérande, Le Croisic, Paimbœuf et La Roche-Bernard), par les pauvres (" tout le peuple de Nantes ") - et certaines institutions - (hôpitaux, prisons, et " autres maisons publiques "). À tous, la tourbe de la Grande Brière Mottière " est aussi nécessaire que le pain qu'ils mangent et que l'air qu'ils respirent, puisqu'ils ne pourraient pas vivre sans feu ".

Quant aux tourbeurs et à ceux qui assurent le commerce de la tourbe, si la ressource venait à disparaître, ils seraient " obligés de s'expatrier, comme des pêcheurs à qui on ôteroit la liberté de prendre du poisson dans la mer ".

22. Voir note précédente. 
Les " chaloupes, chasse-marées et bateaux ", au total 500 à 600 unités d'un tonnage de 6 à 50 tonneaux, dont à Méan 70 chaloupes depuis 20 jusqu'à 50 tonneaux et plus de 100 petits bateaux ", seraient désarmés et leurs équipages sans emploi. Or, ces marins représentent " les deux tiers des habitants de quelques paroisses riveraines de la Brière ", en fait essentiellement de Montoir. Formés sur le tas, dès leur " plus tendre enfance ", ils s'avèrent être " d'excellents matelots qui se sont toujours distingués par leur adresse, leur force et leur valeur ", ce qui en fait les "meilleurs marins de la Bretagne ". Ils peuvent être appelés " au service des vaisseaux de Sa Majesté " et, lors de la " dernière guerre " (guerre de Sept Ans, 1756-1763), la seule paroisse de Montoir a fourni " plus de 800 " (voire " 850 à 900 ", ou pour être précis, " 824 "), " matelots au service de Sa Majesté ", " nombre qu'aucune paroisse maritime n'a jamais égalé ", et qu' "aucune n'a même jamais approché ". En effet, "le pays a fourni cent hommes au roi ", alors que " chacune des autres paroisses de la côte " n'en fournissait que " six ". En cas d'arrêt du commerce de la tourbe, «l'État perdroit une pépinière de marins qui servent si avantageusement la patrie ».

Par ailleurs, en l'absence de tourbe, le bois, déjà rare - d'autant plus que suite à la " disette et la cherté " que vient de subir le pays, il a été abattu bon nombre d'arbres émondables afin de se procurer des ressources pour acheter du pain -, le serait encore plus, les prix flamberaient, ce qui affecterait " considérablement " divers secteurs économiques : la construction navale, les manufactures de verre, de faïence et d'indiennes, ainsi que l'exploitation des mines de fer et la métallurgie, les fers français verraient leurs prix renchérir, alors que leurs prix sont déjà excessifs.

Au final, la Grande Brière Mottière est remarquable par son utilité publique. Elle est un bien public et y porter atteinte est " une entreprise meurtrière, ruineuse pour quarante ou cinquante mille habitants riverains, nuisible à toute une province par ses effets, et préjudiciable à l'État par ses conséquences ".

\section{L'image de la Grande Brière Mottière : une construction "locale " liée à sa défense et fondée sur des stéréotypes}

Élaborée par les opposants à la compagnie de Bray et au seigneur de Saint-Denac, l'image de la Grande Brière est exposée dans des requêtes et des plaidoyers. Elle a été développée en tenant compte de la jurisprudence et en particulier d'un arrêt du Conseil du roi en date du 21 juillet 1764 qui déboute Forestier de Bonabry de sa demande pour dessécher des marais en Bas-Poitou et en Aunis. Cet arrêt, maintes fois invoqué par les opposants au dessèchement - car " les mêmes motifs militoient en faveur des habitants de Montoir et de Saint Joachim, mais qu'ils en avoient encore d'infiniment plus puissants " - précise les conditions à satisfaire afin qu'un dessèchement soit autorisé par le roi. Il impose d'examiner : s'il est techni- 
quement possible, si le résultat à atteindre est avantageux, s'il ne nuit pas aux anciens dessèchements, s'il ne prive par les bestiaux de fourrage et plus généralement les populations de ressources telles que, dans l'affaire Forestier, les roseaux pour la couverture de leurs maisons et le bois blanc pour leur chauffage ${ }^{23}$.

La question de la difficulté technique du dessèchement de la Grande Brière Mottière n'est abordée que par le marquis de L'Estourbeillon - un partisan du dessèchement - qui le dit fort difficile mais non impossible à réaliser. Pourquoi cette question n'est-elle pas abordée par les opposants au dessèchement? Est-ce l'aveu qu'ils n'ont jamais envisagé qu'un pareil projet existait et qu'ainsi ils n'auraient pas à en combattre la faisabilité? Mais sans doute faut-il penser à un positionnement plus tactique; introduire la question de la faisabilité du dessèchement n'aurait pas manqué de faire émerger une série de propositions : dessèchement partiel, mise en place d'une exploitation rationalisée de la tourbe, éventualités que les opposants au dessèchement ne veulent pas aborder.

En effet, ils campent sur une position simple : maintien en état d'un territoire, de ses caractères bien particuliers et de son exploitation traditionnelle. Selon eux, le dessèchement anéantirait ses ressources, tout particulièrement la tourbe qui ne " végéterait " plus et serait détruite par l'incendie, alors que la mise en culture ne saurait créer de ressources équivalentes et appauvrirait le territoire, et que le creusement de canaux le rendait insalubre. Le dessèchement aurait des effets désastreux sur place mais aussi au-delà. La disparition de la tourbe priverait de chauffage surtout des classes populaires et affecteraient gravement des pans entiers de l'économie provinciale, alors que la Marine royale, elle-même, serait affectée en perdant des marins aguerris. Économiquement, socialement, politiquement même, le dessèchement serait une catastrophe et quoi qu'on fasse, " elle ne pouvoit jamais produire des avantages plus importants et plus précieux ${ }^{24}$ ".

Or, un tel projet, "cruel et révoltant ", existe, porté par quelques dessicateurs : " trois ou quatre particuliers ", " hommes cupides et avides ", désireux de "grossir leur fortune ", en provoquant la ruine d'" un très nombre d'individus ${ }^{25}$ ". À un tel discours, une conclusion s'impose : le territoire doit être préservé en son état " naturel ". Bien entendu, une telle défense au nom de la conservation de la " nature " n'a rien à voir avec nos préoccupations actuelles dans ce domaine. Il s'agit alors de défendre l'exploitation traditionnelle du territoire et non pas un biosystème. L'image d'ensemble proposée est une construction bâtie en fonction d'un but à atteindre. Elle

23. Arch. dép. de Loire-Atlantique, C 111, procès-verbal de levée du plan figuratif..., p. 111-112; ibidem, C 112; ibid., E, dépôt, 8, Guérande, DD 1, non daté, requête d'Espivent de La Villeguevray et de Pierre Marie Le Sénéchal.

24. Arch. dép. de Loire-Atlantique, C 111, procès-verbal de levée du plan figuratif..., p. 143.

25. Arch. dép. de Loire-Atlantique, C 112 et ibidem, E, dépôt, 8, Guérande, DD 2 « Moyens précis, procuration des paroisses circonvoisines de la Brière ". 
est défensive mais également offensive : elle est employée par les défenseurs de la cause - des Briérons et leurs relais - afin de convaincre des institutions (États de Bretagne, communautés de ville, généraux de paroisse) et plus largement l'opinion.

Devant différentes juridictions (cour de Guérande, présidial de Nantes, parlement de Bretagne, Conseil du roi), les arguments employés doivent beaucoup aux juristes, procureurs en Cour de Justice (tel Rouaud de La Villemartin agissant également en tant que député de la communauté de ville de Guérande aux États de Bretagne), notaires et procureurs de seigneurie (tel Alain Le Borgne, procureur du général de Saint-André-desEaux), avocats locaux (Chaillon), rennais (souvent consultés) et parisiens en poste à la Cour (Bocquet des Tournelles pour les paroisses). Cependant, ni Espivent de La Villeguevray, seigneur de L'Écurais (en Prinquiau), ni L'Estourbeillon (seigneur du Bois-Joubert en Donges), ni Wlieglen de Luzenbourg (d'origine irlandaise mais possessionné à Donges), dont le rôle est important, ne sont avocats, mais leur statut social (nobles disposant de vastes propriétés pour les deux premiers; en relation avec les milieux d'affaires pour le troisième), leur capacité à développer des argumentaires et à les diffuser sous forme de mémoires les placent au-devant de la scène.

Les « locaux " ont joué un rôle essentiel. Ils sont à l'origine de "l'invention " de la Grande Brière Mottière car l'image qu'ils en proposent fait la part belle aux stéréotypes. Le plus souvent, les ressources du territoire sont réduites à la seule tourbe, et les activités des Briérons, dépeints comme un peuple nombreux, pauvre et laborieux, se limitent à son extraction ou sa commercialisation, vision singulièrement réductrice, et qui gomme notamment les différences entre Briérons des îles et Briérons du pourtour de la Grande Brière Mottière. Territoire et habitants sont placés sous le sceau de la tourbe, ils sont assimilés à une ressource et à sa mise en valeur, et leur importance est appréciée à l'aune de cette ressource.

Cette " invention " s'impose parce qu'elle est en phase avec l'opinion briéronne et l'opinion régionale. Sur place, elle reçoit le soutien des Briérons, soucieux de préserver un territoire sur lequel ils estiment avoir des droits et dont ils tirent leur subsistance grâce à des ressources dont l'eau, pensentils, assure le renouvellement. Leur soutien se manifeste par des actions légales (présence en masse aux assemblées convoquées par les généraux de paroisse), mais également par des actions à caractère illégal : résistances passives, voies de faits, voire " sorte de révolte à main armée ", en 1782, qui paraît avoir provoqué l'arrêt des travaux engagés pour le dessèchement des marais de Donges. L'opinion publique des lieux voisins consommateurs de tourbe est sensible à des affaires que lui font craindre pour son ravitaillement en combustible bon marché. La défense d'un territoire menacé et l'utilité publique de la tourbe à l'échelle de la province conduisent à l'intervention des États de Bretagne, qui, avec quelques réserves, se rallient aux arguments exprimés dans les requêtes des généraux de paroisse. Enfin, à une époque où l'opinion est de plus en plus sensible aux questions sociales, la dénonciation 
de l'action de la compagnie de Bray, présentée comme formée de quelques particuliers avides et cupides, désireux de s'approprier les ressources d'un territoire tout entier au détriment des populations locales et des consommateurs de tourbe dont il est souligné la pauvreté et le caractère laborieux, a cimenté ces diverses opinions.

\section{Le devenir de l'image de la Grande Brière Mottière et la légende noire briéronne (1817-années 1820)}

\section{Le contexte : l'affaire du dessèchement des marais de Donges}

Avec l'ordonnance royale du 2 juillet 1817, qui accorde la concession du dessèchement des marais de Donges à la Compagnie de Bray, s'ouvre un nouveau temps fort de l'histoire briéronne. Cette " affaire des marais de Donges " est marquée par une série de conflits qui se poursuivent jusqu'aux années 1870, ayant trait successivement au dessèchement (achevé en 1825), au partage des terres desséchées (prononcé en 1829), à la délimitation de celles-ci, à la levée de la plus-value, puis aux dommages réclamés par la compagnie de Bray aux communes. Contre cette compagnie, la mobilisation des Briérons est générale et multiforme - instances juridiques, résistances passives, voies de faits, voire véritables émeutes... La tension est parfois si forte qu'au début des années 1820 puis des années 1830, des troupes de ligne casernent sur place.

D'après l'article 4 de cette ordonnance, la Grande Brière Mottière est exclue du dessèchement. Or, pour la période que nous envisageons, son nom est omniprésent. En effet, elle est immédiatement placée au cœur de la querelle judiciaire que soulève cette ordonnance : les opposants à la Compagnie de Bray se réclament d'une Grande Brière Mottière dont certains aspects sont revus à l'occasion.

\section{L'image de la Grande Brière Mottière : invariants et révisions}

Les opposants à la compagnie de Bray proposent une nouvelle délimitation de la Grande Brière Mottière. Elle se fonde sur un passage des lettres patentes du 28 janvier 1784 : celui qui énonce que "tout le peuple commun des paroisses voisines de la Brière " est maintenu " dans la propriété, possession et jouissance commune et publique de ladite Brière mottière [la virgule du texte initial est désormais supprimée, parfois remplacée par un tiret] et terreins contenant des tourbes et mottes à brûler, situés entre et dans lesdites paroisses ". Cette expression, redondante, est interprétée comme désignant deux ensembles : d'une part, la Grande Brière Mottière et, d'autre part, tous les autres terrains tourbeux situés " entre et dans lesdites paroisses " riveraines mais qui, au final, ne forment qu'un seul et même ensemble tourbeux ${ }^{26}$. Aussi la ligne de séparation ordinairement retenue

26. «Enclavé par les communes de Prinquiau, La Chapelle-Launay, Donges, et Montoir, vers l'est; Saint-Nazaire, Saint-André et Escoublac, au sud, Guérande, Saint-Lyphard, 
pour distinguer ces territoires, et que reproduit l'article 4 de l'ordonnance de concession, est-elle déclarée "imaginaire».

Cet argument, qui n'est pas le seul employé, est juridiquement fondamental puisqu'il vise à sanctuariser les marais de Donges. Dès le début, il est asséné par Odilon Barrot, l'avocat des opposants à la compagnie de Bray auprès du conseil d'État ${ }^{27}$. Ce recours à un talent déjà affirmé du barreau change les conditions de la production juridique qui ne fait plus appel comme précédemment à des prises de paroles " locales" et de la "société civile ». Toutefois, cette déconnexion du " local " n'est pas totale puisqu'Odilon Barrot travaille à partir des actes fournis lors des anciennes procédures dont il fait un large usage. Il en résulte une différence de tonalité : le ton est beaucoup plus juridique tant dans la forme que dans l'argumentaire. Cette évolution enregistre la mutation judiciaire intervenue sous la Révolution et l'Empire (effort législatif, codification du droit) ainsi que la réorganisation administrative (disparition des provinces) qui fait que les communes briéronnes se pourvoyant au conseil d'État ne disposent plus de l'appui des États de Bretagne. Dans ces requêtes, les arguments juridiques se veulent péremptoires. Certes les arguments de fait, d'ordre économique et social, ne sont pas omis mais ils restent conformes à l'image traditionnelle du territoire, avec cependant des évolutions.

L'identité de la Grande Brière Mottière était, au travers des pièces de procédure des années 1770-1780, suffisamment établie pour qu'Odilon Barrot n'ait pas à innover. La Grande Brière Mottière dont il fait état est marquée par de nombreux invariants : nature originale, ressources sans cesse renouvelées, importance de la tourbe qui assure la vie des riverains et est un combustible utilisé dans les lieux avoisinants et au-delà.

Le fait d'associer dans un même ensemble Grande Brière Mottière et marais de Donges - désignés sous le seul nom de Brière - conduit à ne plus insister sur la spécificité de la Grande Brière Mottière mais, au contraire, à souligner les aspects communs aux deux territoires : zone humide recouverte par les eaux une grande partie de l'année, présence et exploitation de tourbe (voire de roseaux, analogie de végétation, au moins, sinon seulement, entre Grande Brière Mottière et marais de la Boulaie).

Herbignac et La Chapelle-des-Marais, à l'ouest, et Missillac, Sainte-Reine, Crossac et PontChâteau, vers le nord. La commune de Saint-Joachim est formée de plusieurs îles, au centre ", voir référence note suivante.

27. Mémoire au roi en ses conseils, pour les habitants des communes de Montoir, SaintJoachim, Donges, Saint-Nazaire, Crossac, Besné, Prinquiau, Sainte-Reine, etc., arrondissement de Savenay, département de la Loire-Inférieure contre la compagnie de Bray, Arch. de dép. Loire-Atlantique, 1768, S 1; ibidem, Donges Br in 4e , 1333/7; Bibl. mun. Nantes, 51731, non daté (début 1819); Au roi en son conseil d'État. Mémoire en réplique, pour les communes de Montoir, Saint-Joachim, Donges, Saint-Nazaire, etc., arrondissement de Savenay, département de la Loire-inférieure. Contre des observations faites par son excellence le ministre de l'intérieur dans l'intérêt de la prétendue compagnie de Bray, Arch. de dép. Loire-Atlantique, Donges, $\mathrm{Br}$ in 4e, 1336/6, non daté (été 1819). 
Deux aspects donnent cependant lieu à des précisions. D'une part, est fortement souligné le caractère salubre du territoire qui est mis en rapport avec les propriétés antiseptiques de la tourbe, et, d'autre part, sont décrits les conditions et le processus de sa formation en faisant appel aux idées les plus récentes ${ }^{28}$. Cette insistance nouvelle est en relation avec la législation récente : la loi du 16 septembre 1807 sur les dessèchements affirme qu'ils ne s'imposent que pour les zones insalubres et improductives et la loi du 21 avril 1810 sur les mines et minières fait des tourbières des territoires soumis à une législation spéciale. Aussi, selon ces lois, les marais de Donges ne peuvent-ils être concédés à la compagnie de Bray.

Autre évolution : le recours à la Providence, sauf exception ${ }^{29}$, n'est plus de mise, ce qui traduit l'évolution générale de l'esprit public au lendemain de la Révolution.

Globalement, l'image de la Grande Brière Mottière connaît peu d'évolutions et ses stéréotypes sont confirmés, preuve s'il en est que l'invention de la Grande Brière Mottière lui a conféré une identité extrêmement forte. Toutefois, l'affaire du dessèchement des marais de Donges a été l'occasion d'une réécriture de l'histoire récente du territoire et elle est à la base de la " légende noire " briéronne.

\section{Une révision de l'histoire récente du territoire}

Dans les différentes requêtes présentées pour la défense du territoire, sont rappelés les événements qui l'ont affecté récemment ${ }^{30}$ : afféagement des marais de Donges consenti, en 1771, par le vicomte de Donges à la compagnie de Bray; arrêt du Conseil du roi du 11 juin 1774 ordonnant la levée d'un plan et une enquête préalables au dessèchement; opposition de la population; intervention des États de Bretagne, le 9 février 1775; obtention par la compagnie de Bray en 1779 de la concession du dessèchement; opposition de la population et des États de Bretagne; octroi par le roi, le 28 janvier 1784, de lettres patentes qui, en reconnaissant la propriété des Briérons sur la Brière, en interdisent le dessèchement.

Si le récit est ancré sur des dates et des faits et s'il est cohérent, il n'en donne pas moins aux lettres patentes un sens qu'elles n'ont pas. Selon ce récit, en 1784, en interdisant le dessèchement de la Brière (Grande Brière

28. Voir référence note précédente. Sur la question de la salubrité de la Brière : GALLICÉ, Alain, "La question de l'insalubrité de la Brière au XVIII ${ }^{\mathrm{e}}$ siècle et au début du XIX ${ }^{\mathrm{e}}$ siècle : choc et évolution des représentations d'une zone humide ", Histoire et patrimoine, Association préhistorique et historique de Saint-Nazaire, nº 86, 2016, p. 48-59.

29. Arch. dép. de Loire-Atlantique, 1768 S 1, 21 et 25 juillet 1820, délibération des conseils municipaux de Crossac et de Donges; ibidem, 1767 S 1, sous-préfet de Savenay. Le député de Frénilly fait de la présence de roseaux un véritable don du Ciel (FréNILLY, François Auguste Fauveau de, Notice sur l'arrondissement de Savenay au mois de septembre 1822 Paris, Imp. Boucher, 1823, p. 82).

30. Sur ceux-ci, GALlicé, Alain, «La défense de la Grande Brière Mottière dans la seconde moitié du XVIII" siècle... ", art. cit., pour tout ce développement. 
Mottière et marais de Donges) Louis XVI reviendrait, en raison de l'opposition des Briérons, sur la décision qu'il a prise en 1779. Or, cette présentation est doublement fautive. Elle ne correspond pas à l'enchaînement des faits : la demande de lettres patentes est une démarche autonome dont l'intendant de Bretagne, lui-même, assure qu'elle n'entrave pas l'action de la compagnie de Bray dont les travaux paraissent d'ailleurs interrompus depuis 1782. D'autre part, elle ne correspond pas à la portée géographique des lettres patentes qui ne concernent, quant à elles, que la Grande Brière Mottière.

Cette révision des faits, en dépit des dénégations répétées de la Compagnie de Bray et de l'administration, est acceptée par l'opinion, par la juridiction civile, tant par le tribunal de première instance de Savenay ${ }^{31}$ que par la cour d'appel de Rennes ${ }^{32}$, et elle trouve des échos auprès du préfet de la Loire-Inférieure Villeneuve ${ }^{33}$ et du sous-préfet de Savenay Normand ${ }^{34}$.

Comment expliquer une telle acceptation? D'une part, les faits évoqués sont déjà anciens (les lettres patentes ont été accordées en 1784, soit, en 1817, il y a trente-trois ans) et leur souvenir a dû être fortement troublé par la tourmente révolutionnaire ${ }^{35}$. D'autre part, la formule « entre et dans lesdites paroisses " paraît explicitement confirmer la lecture des opposants à la compagnie de Bray. Or, cette formule est en partie fausse : en effet, la Grande Brière Mottière ne révèle d'aucune paroisse, aussi l'usage d'une formule usuelle du langage juridique s'avère-t-il inadapté et crée la confusion. Par ailleurs, d'un point de vue géographique, la présentation faite correspond à une réalité physique incontestable : la plus grande partie des terrains est tourbeuse. Cette réalité est d'autant plus ressentie que cette tourbe a, de tout temps, donné lieu à une exploitation tant en Grande Brière Mottière que dans les marais de Donges, mais une exploitation de nature différente puisque celle qui est extraite en Grande Brière Mottière est commercialisée alors que celle des marais de Donges, en raison de sa moindre qualité, n'est destinée qu'à être consommée sur place. Toutefois, selon les renseignements recueillis par le sous-préfet de Savenay, à partir de 1817, les zones d'extraction et leur finalité évoluent, les marais de Donges font l'objet d'une exploitation plus importante et destinée, surtout dans les marais de la Boulaie où la qualité de la tourbe

31. Arch. dép. de Loire-Atlantique, 1768 S 2, jugement en date du 29 juillet 1823.

32. Arch. dép. de Loire-Atlantique, 1768 S 2, jugement en date du 23 août 1825.

33. Arch. dép. de Loire-Atlantique, 1767 S 1, 24 octobre 1825, rapport confidentiel du préfet de la Loire-Inférieure au ministre de l'Intérieur.

34. Arch. dép. de Loire-Atlantique, 1767 S 3, 22 et 29 mars 1832. Sa prise de position en faveur des opposants de la Compagnie de Bray lui valut d'être déplacé à Châteaubriant sous la pression de la Compagnie.

35. Cet oubli est également le fait de la Compagnie de Bray qui ne fait pas écho à l'affaire des Équibadeaux, et attribue les lettres patentes du 28 janvier 1784, à une demande de quatre communes désireuses de faire reconnaître leurs droits sur le Grande Brière Mottière, droits reconnus par le Conseil du roi le 4 janvier 1779 aux paroisses de la vicomté de Donges. 
est assez analogue à celle de Grande Brière Mottière, à être commercialisée $^{36}$. De la part des tourbeurs, cette nouveauté a une double portée : juridique et de fait. En effet, elle affirme leurs droits - ils exploitent une terre dont ils estiment avoir la propriété - et l'extraction de la tourbe est un obstacle physique à la réalisation du dessèchement.

Cette réécriture de l'histoire ${ }^{37}$ donne un sens particulier au combat des Briérons contre la compagnie de Bray. Elle le fonde sur des droits de propriété attestés par des "titres" (encore invoqués de nos jours). Or, les dessèchements étant considérés comme une œuvre d'intérêt public, leur réalisation relève du droit administratif et les recours civils ne peuvent en arrêter la marche et le dessèchement est mené à bien. Les Briérons, convaincus que leurs droits sont méprisés, estiment alors justifié leur recours aux voies de fait, voire à des " événements tumultueux ". L'image de Briérons "maîtres chez soi ", soucieux de défendre leur territoire, obstinés et prompts à la révolte, prend ici, sinon son origine, du moins toute sa consistance.

Cette image est empreinte de stéréotypes. Le sous-préfet de Savenay, qui s'exprime le 5 août 1821, c'est-à-dire au lendemain de l'émeute du 31 juillet $^{38}$, affirme que la " nature des habitants n'est pas en cause ", tout au plus les habitants de Crossac et surtout ceux de Saint-Joachim sontils " raisonneurs, tracassiers, insubordonnées ", et d'autant plus virulents que leurs intérêts sont plus "froissés " par le dessèchement que ceux de leurs voisins, jugés plus pacifiques. Selon lui, ces "scènes tumultueuses " qui pourraient être "sanglantes " sont le fait d'" hommes égarés peut-être perfidement excités à la révolte, mais qui au fond sont dévoués au gouvernement du roi ${ }^{39}$ ". Volonté de se rassurer de la part d'un administrateur que la révolte a traumatisé? Sans doute, mais seulement en partie, puisqu'il persiste dans ses convictions, en affirmant, le 29 août 1823, que les Briérons sont " naturellement bons et tranquilles ${ }^{40}$ ». Une telle opinion n'est pas en accord avec la légende noire briéronne.

36. Arch. dép. de Loire-Inférieure, 1767 S 1, 29 août et 2 septembre 1821, Voir encore : FRÉNILLY, François Auguste Fauveau de, Notice sur l'arrondissement de Savenay..., op. cit., p. 80-81; rapport confidentiel du préfet de la Loire-Inférieure au ministre de l'Intérieur, Arch. dép. de Loire-Inférieure, 1767 S 1, 24 octobre 1825; Emmanuel Joseph Olivaud, maire de Montoir, Bibl. mun. Nantes, ms. 1748, p. 20.

37. Encore acceptée en particulier par GuIHAIRE, Albert, La Brière..., op. cit., p. 183-184 et plus récemment par LE MAREC, Yannick, "Les émeutes de la Brière dans la première moitié du XIXe siècle ", dans ANTOINE, Annie, MIschI, Julian, Sociabilité et politique en milieu rural, Rennes, PUR, 2008, p. 51-63.

38. Ibidem.

39. Arch. dép. de Loire-Atlantique, 1767 S 1, 5 août 1821.

40. Arch. dép. de Loire-Atlantique, $1766 \mathrm{~S} 1$. 


\section{La légende noire briéronne}

La légende noire briéronne s'exprime dans un article intitulé « La Brière ${ }^{41}$ ", signé Ludovic. Sous ce pseudonyme se cache Ludovic Chapplain, le gérant responsable de la revue Lycée armoricain, éditée par Camille Mellinet ${ }^{42}$.

L'image qu'il donne de la Grande Brière Mottière est, d'entrée, totalement négative : " Ici, plus de champs, plus de culture, la civilisation s'arrête, et la Bretagne disparaît [...] c'est l'immensité de ses landes, mais sans leur imposant aspect et leur lointain horizon [...]. Autour de nous, une nature âpre, sauvage, étrange, sous vos pieds, une terre brune, fangeuse et mobile; au loin un cercle noir qui vous entoure, et comme un long voile de deuil semble ceindre cette misérable contrée... " Dans un tel environnement " que demander ici à l'imagination? [...]. Ses prismes sont brisés et ses fantômes évanouis [...]. Plus de poésie dans le cœur, de charme dans les souvenirs, ce noir horizon est là, devant vous, comme une pensée pénible, inévitable... ".

Certes, "à travers cette vapeur qu'exhale le sol humide " dans ces " noires savanes, il y a des bourgades, des hommes, des familles, un peuple qui vit en société et se dit français...". Cette constatation, somme toute surprenante au vu de l'environnement, conduit immédiatement l'auteur à s'interroger : "Français! [...] et le moyen d'y croire [...]. N'est-ce pas plutôt une terre étrangère rejetée bien loin au-delà des mers? [...]. Car de la France, autour de vous, pas la plus légère trace, pas un faible reflet [...], pas un bouquet d'arbres qui rappelle ses frais paysages, pas le moindre murmure qui rende le bruit de la vie..."

De ce triste constat témoignent diverses descriptions. Et d'abord, celle du cadre de vie du Briéron. Sous le toit de chaume d'une " cabane ", toute emplie d'une " fumée âcre et noire " provenant de la combustion de la tourbe, se distinguent " aux quatre coins du taudis, quelques grabats recouverts de sacs de laine, sur lesquels s'entassent, pêle-mêle, hommes, femmes, enfants et valets; puis les têtes des bestiaux qui se montrent à travers les barreaux de la cloison à claire-voie ». Dans ce cadre, des "fantômes [...] se meuvent lentement ". Leurs portraits sont brossés. Celui du chef de famille : "Figure jaune et sèche, morne et stupide, que recouvre à moitié une barbe épaisse et qu'abrite un bonnet de laine brun, puis vient un corps immobile enveloppé de sombres vêtements. " Puis celui d'une autre créature " qui ressemble à l'ébauche informe d'un sculpteur; et cette peau noire et ridée, cet œil fixe?

41. Ludovic, "Esquisses bretonnes : la Brière ", Revue de l'Ouest, 1, 1829-1830, p. 161170. En 1830, le Lycée armoricain fusionne avec la Revue de l'Ouest fondée en 1828, et les deux revues sont livrées en même temps. En 1831, le titre devient Revue de l'Ouest, ancien Lycée armoricain.

42. Ludovic Chapplain, qui fut plus tard bibliothécaire de la ville de Nantes et archiviste du département, a utilisé également d'autres pseudonymes, tels ceux de Budic l'Armoricain ou du Flâneur Breton, et, sous ce dernier, il publie, dans les premiers numéros du Lycée armoricain des chroniques sur la vie et les mœurs à Nantes dont la forme est " vivante et spirituelle " (CHANTREAU, Alain, "Camille Mellinet, sa famille et son temps", Bulletin de la Société archéologique et historique de Nantes et de la Loire-Inférieure, t. 120, 1984 , p. 142). 
Que pensez-vous que ce puisse être? Vous ne devinez pas? Tout cela forme l'ensemble de la physionomie de l'épouse; oui, vous pouvez m'en croire, c'est véritablement une femme, et telle que vous la voyez, elle fait partie de ce sexe qu'ailleurs on adore, on divinise "... Quant aux enfants : "L'influence du pays pèse déjà sur eux de tout son poids, la fleur de la jeunesse s'est fanée chez eux, et les voilà mornes, blêmes, impassibles comme leur père. "

La conclusion s'impose, nette et tranchante : on se croirait " chez une famille du Kamschatka ou du pays des Hottentots "... Destiné à rendre compte de l'étonnante altérité des Briérons, le recours à des comparaisons exotiques se retrouve encore dans l'évocation des "villes de la Basse-Égypte, durant les inondations du Nil ", pour évoquer les îles qui parsèment la Grande Brière Mottière, et celle des embarcations de Venise pour évoquer celles de Brière; mais Ludovic a soin de souligner la dissemblance des deux lieux qui se lit " avec des toits de chaume pour palais, avec des barques grossières au lieu de gondoles élégantes ". L'exotisme est encore convoqué avec l'indication de possibles mirages, analogues à ceux des " déserts d'Afrique et d'Égypte ".

Ludovic poursuit son propos en insistant sur l'anachronisme du Briéron en le montrant insensible au spectacle de la beauté et de l'animation de la Loire ainsi qu'aux attraits de Nantes qu'il fréquente pourtant " dix fois par an ". Bref, « il n'a pas fait un pas dans la civilisation; tel il était il y a quatre siècles, tel il est encore "...

Ludovic évoque également la formation de la tourbière, l'extraction et le commerce de la tourbe. Le Briéron exploite cette terre particulière où, par une "étrange prévoyance ", la nature " pour protéger son ignorance et lui épargner la peine d'ensemencer pour vivre, a placé à ses pieds des trésors faciles à exploiter. Cette terre noire qui attriste vos regards, voilà sa richesse; et il ne l'échangerait pas pour la poudre d'or du Nouveau Monde ". Elle fait vivre ces habitants en assurant à la " ménagère " l'argent dont elle a besoin, et aux enfants le « vieux lard et [...] la soupe à la graisse qui vont, pendant quelques jours, varier agréablement leurs maigres repas composés de pain sec et de pommes de terre".

La Grande Brière Mottière assure la vie des Briérons et il y a symbiose entre elle et la population qui l'exploite. Le Briéron est donc une sorte d'émanation de la Grande Brière Mottière et sa vie est une allégorie de ce territoire qui lui offre une ressource " primaire " pour subsister, et qui est l'objet d'un commerce tout aussi " primaire " quant à son organisation. L'existence du Briéron est tout aussi " primaire ". Le mariage n'est qu'une simple " spéculation qui n'interrompt en rien le cours monotone de la vie ", et la cérémonie qui l'accompagne est expédiée rapidement et sans joie. La vie familiale se résume en une formule : " une femme pour tremper la soupe, des enfants pour recueillir la tourbe" ; et la vie sociale en une autre : " accumuler les profits qu'il retire de son commerce, voilà sa règle de vie, voilà tout son bonheur ". " La mort n'est qu'un simple passage obligé et accepté, aussi n'est-il rien tenté par ses compatriotes quand un Briéron tombe à l'eau, ils se contentent d'énoncer : "c'est son bon ange qui l'appelle vers lui"; et, muets, les bras croisés, ils se 
soumettent à la volonté du ciel en murmurant quelques prières ". Le deuil est tout aussi " primaire " : il est célébré par des " hurlements sauvages ", et c'est "à qui criera le plus fort pour honorer le défunt ". Dans ce monde " primaire " où les mirages ne sont pas absents, le Briéron est en proie aux superstitions. Les " flammes bleues qui scintillent parfois sur la Brière " sont identifiées comme étant des " esprits malins prêts à égarer le voyageur et à l'entraîner dans les fondrières ", et les vapeurs blanches sont reconnues comme "les ombres de leurs parents, de leurs amis ". Quant aux événements dramatiques qui pèsent sur les hommes, leurs bestiaux ou leurs récoltes, ils sont attribués à des sorciers jeteurs de sort qui vivent en marge de la communauté.

Cette description qui affecte la forme du récit d'un voyageur soucieux de rendre compte de ce qu'il observe autour de lui est en totale contradiction avec ce que rapporte le député Frénilly ${ }^{43}$ ou le docteur Olivaud ${ }^{44}$. Aussi le sens de cette charge doit-il être recherché. Il est à trouver dans l'évocation par Ludovic de la question du dessèchement des marais de Donges. Il met en exergue l'ambition des dessicateurs qui, en assurant la mise en culture et l'assainissement du marais, affirment apporter la richesse au pays et le bonheur aux populations ${ }^{45}$, ambition hautement affirmée et partagée par l'administration ${ }^{46}$, et plus largement par l'opinion " éclairée ${ }^{47}$ ".

43. FrÉNILly, de, François Auguste Fauveau de, Notice sur l'arrondissement de Savenay..., op. cit., p. 74-83, il dépeint des Briérons habillés de « bure noire ", " barbe hérissée, la figure enfumée et sauvage, encadrée de deux rivières de cheveux noirs ", et comme formant une "nation qui semble être sortie de la tourbe bretonne". Les Briérons sont " rudes, impatients et guerriers ", mais " bretons, c'est-à-dire religieux, probes et fidèles; religieux dirait tout : qui craint Dieu, aime son prochain et ses maîtres". Il souligne encore la qualité de la mise en valeur des îles " corbeille ravissante de richesse, de vie et de végétation ", et des maisons, "toutes propres, la plupart bien bâties, quelques-unes toutes neuves ".

44. En réponse à un questionnaire du comité de salubrité de Nantes, le docteur Emmanuel Joseph Olivaud, maire de Montoir, insiste sur la bonne santé des Briérons, leur alimentation variée et les progrès de l'habitat : désormais construites au même niveau que le sol, les chaumières les plus récentes sont dotées d'une fenêtre; un feu de tourbe est allumé en permanence pendant " la saison froide et pluvieuse ", ibidem, 5 M 86/3; GALlicé, Alain, "La question de l'insalubrité de la Brière... ", art. cit., p. 48-59. Cependant, le 31 juillet 1821, le sous-préfet de Savenay fait état du fait que les soldats envoyés pour rétablir l'ordre ont préféré, à Besné, coucher sur la paille dans une grange du presbytère plutôt que d'habiter les " demeures étouffantes et malpropres des paysans " (Arch. dép. de Loire-Atlantique, $1767 \mathrm{~S} \mathrm{1,} 12$ août 1821).

45. Par exemple, Arch. dép. de Loire-Atlantique, 1768, S 1, octobre 1817, texte envoyé par la Compagnie de Bray (relayé par l'administration préfectorale) sous forme de placards destinés à être affichés dans les communes et vantant les bienfaits du dessèchement : "Autour de ces eaux croupissantes et pestilentielles, qui annoncent aujourd'hui la misère, les villages vont s'accroître, les troupeaux se multiplier. Cette inondation immense, dont l'aspect attristait, a fixé les regards de notre bon roi, aussitôt elle doit disparaître, et faire place à un sol fécond, créateur, à l'une des plus riantes et des plus riches contrées. "

46. Par exemple, Arch. dép. de Loire-Atlantique, 1738 S 1, 13 avril 1819, lettre du ministre de l'Intérieur au garde des Sceaux; ibidem, 1768 S 2, non daté (1825), exposé de l'affaire du dessèchement de Donges.

47. Voir, par exemple, le discours de Freteau, président de la Société académique du département de la Loire-Inférieure, prononcé lors de la séance publique de la Société, tenue le 3 août 1820, Bulletin de la société académique, 1820, p. 35-38. 
Mais les Briérons ne l'ont pas entendu ainsi : " alors ce peuple que l'on croyait mort à toute sensation s'éveilla, terrible ". Par leur révolte, modernes Mazaniello ${ }^{48}$, les Briérons - donc la violence, signifiée par les mots " insurrection ", " révolte à main armée ", " guerre ", " lutte sanglante ", " victimes ", est exagérée - affirment les liens consubstantiels et fusionnels qui les unissent à la Brière, car " qu'importait au Briéron, et ces richesses promises, et cette métamorphose qu'il ne demandait pas [...] La terre noire et fangeuse qu'on voulait lui enlever faisait vivre sa famille; elle avait nourri ses pères, elle était aussi belle à ses yeux que les plus riants paysages, aussi riche que les plus fertiles coteaux [...]. Le bien présent était tout pour lui : qui lui assurait que l'avenir serait meilleur?"

Le texte s'achève sur une phase qui pourrait paraître teintée d'ironie : " On continua à se disputer ce marais ni plus ni moins que si c'eût été un paradis terrestre. " En fait, la phrase révèle le sens du récit. Elle exprime que les uns et les autres se font des représentations idéalisées de leur monde et qu'elles sont antagonistes, incompatibles et irréductibles l'une à l'autre. La Brière telle que la voient les Briérons ne peut que disparaître sous la pelle et la pioche des dessicateurs, et, pour la conserver, ils s'opposent à leurs travaux par des moyens de droit ou encore par la force. Mais dans cet affrontement qui engage l'existence du monde traditionnel briéron, la lutte ne se développe pas seulement dans les prétoires et sur le terrain, elle se situe aussi au niveau des représentations. Les dessicateurs par un double jeu de miroirs déformants font, d'une part, de ce monde traditionnel, un monde de misère, d'ignorance, que son altérité rend étrange, exotique et anachronique et qui, de ce fait, n'a plus de raison d'être. Et, d'autre part, un autre miroir déformant fait du monde que ces modernes démiurges promeuvent un monde d'abondance, de progrès, qui ferait entrer les Briérons " dans l'histoire ". Aux yeux des Briérons, ces représentations sont inacceptables : le monde traditionnel leur assure la vie, certes une vie sans doute fruste mais analogue à celle de leurs pères et indépendante, alors que le monde qu'on leur promet signifie leur dépossession et ne paraît leur offrir aucune certitude d'une vie meilleure.

En paraphrasant une formule de l'auteur, - celle où il évoque « le droit de bouleverser leur pays pour faire leur bonheur en dépit d'eux-mêmes " -, on peut énoncer la morale qu'appelle cette histoire : on n'a pas le droit bouleverser un pays et de faire le bonheur de sa population contre son gré.

Ce sont ces visions croisées que Ludovic donne à lire; mais d'une lecture rapide, au premier degré, se dégage un portrait éminemment défavorable des Briérons ${ }^{49}$. Cette vision s'est imposée. Pourquoi? La raison essentielle est que les milieux éclairés la partagent tant la réputation des zones humides est alors exécrable et que l'attitude des Briérons leur apparaît être irrationnelle. À ce propos, les témoignages sont nombreux, contentons-

48. Nom donné aux Napolitains révoltés contre les Espagnols en 1646.

49. Le texte a fait l'objet d'une réédition ultérieure qui en retranche le début et surtout la fin et donc dénature le sens en ne retenant que la charge anti-briéronne. 
nous d'en citer deux émanant de l'administration préfectorale : le 16 juillet 1832, le sous-préfet de Savenay évoque le sort de ses habitants « entourés d'eau, sans communication ", restés " étrangers au progrès de la civilisation " et formant " un peuple à part ", hostile à toute " innovation " et attaché "à de vieilles traditions qui ne sont le plus ordinairement que de vieux préjugés ${ }^{50}$ "; le 8 août 1832, le préfet de la Loire-Inférieure fustige les « habitudes invétérées des habitants misérables et ignorants de ces communes " incapables de concevoir le " sort nouveau et plus heureux qu'on veut leur faire connaître ${ }^{51}$ ". D'autre part, beaucoup parmi les plus compréhensifs envers les Briérons finirent, en raison de la longueur du conflit, par perdre patience et se lasser du continuel refus de compromis des Briérons. Et puis encore, et peut-être surtout, les Briérons n'hésitèrent pas à l'utiliser pour leur cause comme le montre un épisode de l'année 1841. Pour éviter des sanctions, le maire de Montoir plaide la clémence de l'administration en ces termes : "Nos communes sont déjà trop malheureuses. " Le 2 septembre 1841, le sous-préfet de Savenay transmet ce courrier au préfet, et, reprenant la pensée du maire, afin d'appuyer la requête du maire, il s'exprime ainsi :

" Cette population est ignorante et presque sauvage, la civilisation qui a fait tant de progrès dans les autres parties de la France, n'a pu pénétrer dans les marais de Saint-Joachim, où l'empire de la routine et de l'habitude l'emporte sur le respect qui est dû aux lois; mais il est aussi vrai, toutefois, qu'elle est misérable et que la tourbe est sa seule ressource ${ }^{52}$."

Dans l'affaire, il s'agit pour les autorités locales d'éviter des sanctions, aussi disent-elles aux autorités supérieures ce qu'elles veulent entendre. Elles évoquent l'opinion que l'on a de l'extérieur de la Brière, celle de la légende noire, opinion admise, sinon partagée par l'administration : celle de Briérons, attardés, misérables, dépendants de la seule tourbe pour vivre, toujours prêts à intervenir pour défendre leur territoire et sa ressource.

La seconde moitié du XVIII ${ }^{\mathrm{e}}$ siècle voit l'invention de la Grande Brière Mottière. Entre 1817 et 1830, cette vision du territoire est confortée et précisée. Elle est intimement liée à la tourbe avec laquelle les Briérons ont un lien si fort que l'idée s'impose qu'ils sont issus de la tourbe, idée que le mythe de Cadmus vient magnifier ${ }^{53}$. Cette construction s'est nourrie de la production d'écrits issus du territoire et destinés à défendre la Grande Brière Mottière lugée menacé. Elle est valorisante et insiste sur ses ressources, son utilité pour les riverains et les environs, son caractère sain, mais fragile qu'il faut en conséquence préserver. Cette défense du territoire est encore fondée sur les droits que les Briérons affirment avoir sur le ter-

50. Arch. dép. de Loire-Atlantique, $1767 \mathrm{~S} 3$.

51. Arch. dép.de Loire-Atlantique, 1767 S 3.

52. Arch. dép. de Loire-Atlantique. $1705 \mathrm{~S} \mathrm{1;} \mathrm{ibidem,} 1706 \mathrm{~S} 1$.

53. FRÉNILLY, de, François-Auguste Fauveau de, Notice sur l'arrondissement de Savenay..., op. cit., p. 79, évoque une "nation qui semble être sortie de la tourbe bretonne, comme les enfants de Cadmus de la terre Thébaine ". 
ritoire qu'ils font valoir au prix d'arrangements avec l'histoire en faisant d'un simple mandement ducal de 1461 des lettres patentes et en étendant la portée géographique de celles de 1784 aux marais de Donges.

Face à cette image visant à maintenir en l'état un monde traditionnel, entre 1817 et les années 1830, apparaît une autre invention, celle de la légende noire briéronne. Ici la tonalité est totalement dévalorisante. Venue de l'extérieur, destinée à montrer ce que, par opposition, le dessèchement des marais de Donges apporterait de progrès et de mieux-vivre, elle s'impose, portée qu'elle est par les milieux favorables au dessèchement, mais aussi instrumentalisée par les Briérons au profit de leur cause.

Le fait que l'impact de ces inventions forgées par les uns et les autres ne soit de nos jours pas encore entièrement dissipé montre toute la force de ces représentations. Cependant, leur bilan doit aussi être apprécié à l'aune des objectifs pour lesquels ces inventions ont été forgées. En 1825, certes, la Grande Brière Mottière reste en l'état, mais elle n'est pas directement menacée, alors que les marais de Donges sont desséchés. Cependant, à long terme, pour les Briérons, le bilan n'est pas négatif : les pressions sur la zone humide restant fortes, les représentations du territoire et des hommes auront alors leur part dans le positionnement des acteurs et elles ont contribué à faire échouer un à un tous les projets ou les tentatives de dessèchement ${ }^{54}$.

54. GALliCÉ, Alain, « Pourquoi et comment la zone humide de la Grande Brière Mottière a-t-elle été préservée? Indivision des terres, exploitation de la tourbe et commission syndicale jusqu'en 1921 ", Bulletin de la société archéologique et historique de Nantes et de Loire-Atlantique, t. 145, 2010, p. 177-232. 


\section{RÉSUMÉ}

Durant la seconde moitié du xviiie siècle, l'affaire des Équibadeaux et le projet de dessèchement des marais de Donges font craindre que la Grande Brière Mottière ne soit desséchée et que ses habitants ne perdent ainsi ses ressources. Pour défendre le territoire, dans les années 1770-1780, des hommes issus de la société locale élaborent une image du territoire centrée sur la tourbe et son exploitation par les Briérons. Cette représentation valorisante et fortement empreinte de stéréotypes est confirmée et enrichie entre 1817 et les années 1830 à la suite de l'affaire des marais de Donges. En opposition, la forte opposition des Briérons au dessèchement des marais de Donges fait naître la " légende noire " briéronne, fortement dévalorisante et faisant appel encore plus largement aux stéréotypes. La force de ces inventions, en termes de représentations, est telle qu'elles ont pesé sur l'histoire de la Grande Brière Mottière (dont le nom s'impose progressivement), et que leur impact ne s'est pas totalement, encore de nos jours, dissipé.

\section{ABSTRACT}

During the second half of the eighteenth century, the affair of Équibadeaux and the project of draining the Donges marshes threatened to dry up Grande-Brière Mottière and thus endangered the livelihood of local inhabitants who relied on its resources. To defend the area in the years 1770-1780, local people sought to portray themselves as being dependent on peat and its commercialisation. This positive representation, strongly influenced by stereotypes, was further confirmed and strengthened between 1817 and the 1830s following the affair of the Donges marshes. In contrast, the strong opposition of Brierrons to the draining the Donges marshes created a "Brieronese black legend " that resulted in a much more negative portrayal that appealed even more widely to stereotypes. The strength of these fabrications, in terms of representation, was such that they had a profound impact on the history of Grande-Brière Mottière (as it became known) - so much so that their influence can still be perceived today. 